\title{
Comportamiento del rendimiento y frecuencia en el tamaño de la semilla F1 de maíz (Zea mays L.) y su relación con la pérdida de hojas en el despanojado de líneas parentales
}

\author{
Performance of Yield and Frequency of Seed F1 Maize (Zea mays L.) \\ Size and its Relationship with Loss of Leaves on Parental Lines \\ of Detasseling
}

Comportamento de desempenho e freqüêencia no tamanho da semente F1 de milho (Zea mays L.) e sua relação com a perda de folhas na descompactação de linhagens parentais

Julio Quiroga-Cardona' ${ }^{1}$

1 I.A, MSc, Investigador Científico I, Centro Nacional de Investigaciones de Café, CENICAFÉ, Sede Planalto,

km. 4 vía Chinchiná-Manizales. Manizales (Caldas) - Colombia

Email: julio.quiroga@cafedecolombia.com

Recibido: 20 de mayo de 2016

Aceptado: 21 de mayo de 2019

\begin{abstract}
Resumen
En el proceso de producción de semilla híbrida de maíz es requerimiento que se realice el despanojado o eliminación de la inflorescencia masculina en las líneas maternas. Durante este proceso se produce la pérdida de las hojas superiores y que acompañan la panoja, esta pérdida en área foliar afecta negativamente la producción de semilla y la frecuencia de granos planos y redondos en los calibres comerciales. En el presente trabajo se analizó el comportamiento de una línea materna, progenitora de un híbrido comercial de maíz convencional de endospermo blanco, sometida a distintos tratamientos relacionados con la pérdida de área foliar al momento de la eliminación de la inflorescencia masculina. Se estimó la producción de semilla por hectárea y la frecuencia de formación de granos F1 planos y redondos en los calibres aceptados comercialmente en el mercado. Los resultados demuestran que cuando hay pérdida de hojas en la planta de maíz al momento de eliminar la panoja, fisiológicamente se afecta su rendimiento, no obstante, se puede tolerar la pérdida de una a cuatro hojas sin que ello represente diferencias estadísticas en el rendimiento. Las frecuencias de tamaño en granos planos y redondos en las semillas F1 de tipo comercial no se vieron afectadas.
\end{abstract}

Palabras clave: autopolinización, despanojado, híbrido, panoja, polinización.

\begin{abstract}
In the production process of maize hybrid seed is requirement to do detasseling or removing the male inflorescence in maternal lines. During this process the loss of the upper leaves and accompanying the panicle occur, this leaf area loss negatively affects seed production and flat and round grains frequency in commercial calibers. This work analyses the be-
\end{abstract}


havior of a maternal line, progenitor of a commercial hybrid of conventional maize with white endosperm. This line was subjected to various treatments related with the loss of leaf area when removing the male inflorescence. The behavior of seed production and frequency of formation of flat and round F1 grains in the gauges that are commercially accepted in the market were estimated. The results showed that when there is loss of leaves on the maize plant when removing the panicle, physiologically the development based on yield is affected, however it can tolerate the loss of one to four leaves without representing statistical differences in yield. Similarly, the size frequencies of flat and round grains in F1 commercial seeds were not affected

Keywords: self-pollination, detasseling, hybrid, panoja, pollination.

\section{Resumo}

No processo de produção de semente hibrida de milho é requisito o despendoamento, ou remover a inflorescência masculina em linhas maternas. Durante o processo se produz a perda das folhas superiores que acompanham a pendão, esta perda da área foliar afeta negativamente a produção de sementes e frequência de grãos planos e redondos em calibres comerciais. Neste trabalho avaliou-se o comportamento de uma linha materna, progenitor de um híbrido comercial de milho convencional de endosperma branco e que foi submetido a vários tratamentos relacionados com a perda da área foliar na hora da remoção da inflorescência masculina. Avaliou-se o comportamento da produção de semente por hectarea e a frequência de formação de grãos F1 planos e redondos nos calibres que estão comercialmente aceitos no mercado. Embora os resultados mostram que, indiscutivelmente, quando há perda de folhas nas plantas de milho quando remover o pendão, fisiologicamente o desenvolvimento é afetado em função do desempenho, no entanto pode tolerar a perda de 1 a 4 folhas sem representar diferenças estatísticas sobre o desempenho. No entanto, as frequências de tamanho em grãos planos e redondos na F1 de sementes do tipo comercial não são afetados.

Palavras-chave: auto-polinização, despendoamento, híbrido, pendão, polinização.

\section{Introducción}

En la producción de semilla de híbridos de maíz, el despanoje o desespigamiento, es una de las prácticas que requiere gran atención y cuidado, ya que de su correcta realización depende el adecuado cruzamiento entre los progenitores para la obtención de semilla de híbridos con buena calidad genética y por ende la heterosis definida por el mejorado.

Desde otro punto de vista, el despanojado es la técnica de eliminar la panoja de las plantas que participan como hembras en la formación de híbridos de maíz. De esta manera se asegura que la semilla que se recolecta en campo al momento de la cosecha corresponda a un híbrido F1 y no a una autopolinización de las plantas que participaron como hembras en los cruzamientos definidos. El despanojado adecuado mantiene la pureza genética y evita contaminaciones con polen que genera autofecundaciones cuando no se hace oportunamente (Espinosa-Calderón, et al., 2010).

La eliminación de la panoja debe hacerse antes que la planta inicie su proceso de liberación del polen, lo cual conlleva a que en este proceso se pierdan de una a tres hojas y dependerá de la etapa en que se haga la eliminación de las estructuras reproductivas masculinas. Las etapas de eliminación pueden ser antes que la panoja salga o se encuentre aun cubierta por las hojas adyacentes o después que ésta haya emergido totalmente, pero antes que inicie la liberación del polen. Las hojas que se pierden son las que están más cerca- nas a la panoja o estructura reproductiva masculina. Sin embargo, cuando se cuenta con líneas maternas que presentan el pedúnculo de la panoja largo y que es una característica deseable, la pérdida de hojas al momento del despanoje es menor. Es por esta razón que la labor de eliminación de la panoja es una de las actividades más costosas en la producción de semillas de maíz, éstos costos se pueden disminuir con la utilización de líneas maternas andro-estériles.

La androesterilidad facilita la producción de semilla y favorece el mantenimiento de la calidad genética. Del mismo modo, esta característica genética permite disminuir los costos de producción porque se omite el despanoje que representa una actividad fundamental para lograr la calidad e identidad genética del híbrido correspondiente, (Espinosa-Calderón, et al., 2010). Adicionalmente, favorece un menor precio de semilla, porque se prescinde de esta práctica (Tadeo-Robledo, et al., 2003) citado por (Espinosa-Calderón, et al., 2010). No obstante, la androesterilidad dejó de utilizarse durante en la década de los 70`s, debido a su relación genética con la susceptibilidad a la enfermedad del tizón foliar causada por el hongo Helminthosporium maydis raza $\mathrm{T}$, el cual ocasionó una epifitia en gran parte de la faja maicera de los Estados Unidos (Tadeo-Robledo, et al., 2003). Todos los híbridos de maíz que contenían el gene para la esterilidad citoplásmica presentaron susceptibilidad y se perdió casi el $15 \%$ de todo el maíz producido en los Estados Unidos en 
aquel año. El valor monetario de las cosechas perdidas se estimó en mil millones de dólares (Agrios, 2012).

Con el fin de determinar las mermas en rendimiento de una línea materna, que participa cómo progenitora de un hibrido comercial de maíz de cruza simple, se realizó este trabajo buscando identificar cuál es el número máximo de hojas que se pueden perder en el despanojado, sin afectar el rendimiento de semilla híbrida de maíz, proyectando al futuro una metodología para el despanoje mecánico que permita perder en el proceso un número determinado de hojas, sin que esto influya drásticamente en los rendimientos y que la producción de semilla comercial de híbridos de maíz no se vea afectada.

Cuando se elimina sólo la panoja, se presenta un incremento en la producción de semilla de la línea hembra, esto se explica en el rompimiento de la dominancia apical que favorece la producción y calidad de semilla (Espinosa-Calderon, et al., 1995), citado por (EspinosaCalderon, et al., 1999). No obstante, es deficiente la información en referencias bibliográficas sobre la influencia de la pérdida de hojas en el despanojado en la frecuencia de aparición de granos de tipo plano y redondo en semillas de maíz hibrido de tipo comercial. En este trabajo se determinó una aproximación a este comportamiento.

\section{Materiales y métodos}

El experimento se llevó a cabo durante el segundo semestre del año 2013, en la finca experimental Algarrobo, ubicada en el corregimiento de Rozo, localizado en departamento del Valle del Cauca - Colombia. Se empleó un diseño experimental bloques completos al azar con siete repeticiones. Se evaluó una línea materna tropical (isugu-94-F2-MZ1)-4-\#), productora de un hibrido comercial (SEM1430). La densidad poblacional empleada fue de 62.500 plantas ha ${ }^{-1}$.

Las características climáticas de la zona donde se realizó la evaluación fueron tomadas de consultas a la base de datos de la Red Meteorológica Automatizada - RMA de Cenicaña (Centro de Investigación de la Caña de Azúcar de Colombia, 2013) estación meteorológica de referencia Rozo. Se tomaron como referentes los datos registrados por la estación desde el año 1993 hasta 2013. Siendo la temperatura mínima de 7.1 , media 23.5 y máxima de $34.9{ }^{\circ} \mathrm{C}$. La humedad relativa, mínima 0.0 , media 77.6 y máxima $100.0 \%$. La precipitación en el área de estudio fue de $0.0 \mathrm{~mm}$ como dato mínimo, precipitación media $0.1 \mathrm{~mm}$ y precipitación máxima presentada $52.4 \mathrm{~mm}$.
Los tratamientos evaluados fueron siete: (1) sin despanojar (SinDesEs), (2) despanojado normal, como se hace comúnmente en el campo (NormalB), (3) corte de la panoja +1 hoja $(E s p+1 \mathrm{Hoj})$, (4) corte de la panoja +2 hojas (Esp+2Hoj), (5) corte de la panoja +3 hojas $($ Esp +3 Hoj), (6) corte de la panoja +4 hojas (Esp+4Hoj) y (7) corte de la panoja +5 hojas (Esp+5Hoj).

El corte de la panoja y hojas se realizó de forma manual con una tijera de poda, las hojas involucradas en el corte fueron las que se encontraban más próximas a la panoja, quedando en promedio 3 hojas por encima de la mazorca. El tratamiento del despanojado normal como se realiza comúnmente en la práctica y de forma manual, se realizó teniendo en cuenta el método empleado por las personas que generalmente se contratan para realizar esta labor en lotes comerciales destinados a producir semilla. En esta labor, por lo general siempre se pierde más de una hoja, sin ser esta pérdida homogénea.

Las unidades experimentales estuvieron constituidas por 6 surcos de 5 metros de largo cada uno. En campo se usó un arreglo poblacional de dos surcos de línea paterna por cuatro surcos de línea materna, espaciados a 20 centímetros entre plantas y 80 centímetros entre surcos. La parcela útil fue los cuatro surcos centrales desechando las plantas de los bordes. La preparación del terreno se realizó con maquinaria agrícola, un pase de arado y dos pases de rastra y el establecimiento del ensayo se realizó en forma manual; la fertilización se realizó manualmente y de forma fraccionada en dos aplicaciones, la primera transcurridos los primeros 15 días después de la germinación ( 6 bultos de un fertilizante de síntesis química (12-24-12), por hectárea, 1 bulto de fertilizante de elementos menores por hectárea) y la segunda, un mes después de la primera aplicación (6 bultos de Urea y 2 bultos de KCL por hectárea). Los requerimientos nutricionales del cultivo, fueron según los requerimientos para el cultivo en la zona (García-Montealegre, 2008) y a los resultados obtenidos del análisis químico de suelo, obtenido días previos a la siembra. El requerimiento hídrico fue suplido mediante la aplicación de riego por gravedad, riego por surcos y según se veía la necesidad de humedad en el suelo, de igual modo los controles fitosanitarios y manejo de malezas se realizaron según la necesidad que se presentó en campo. Los controles de malezas en los primeros estados del cultivo, mientras cerraba calles con herbicidas selectivos y el control de insectos Spodoptera frugiperda (Lepidoptera: Noctuidae), con inhibidores de quitina.

Las variables evaluadas fueron: producción de semilla la línea materna, sometida a los diferentes tratamien- 
tos, y la frecuencia de semilla híbrida teniendo en cuenta los distintos calibres para selección y comercialización. Para la determinación de la frecuencia de semillas respecto al calibre, se empleó 1.0 kilogramo de semilla tomado al azar del total obtenido de las unidades experimentales y analizado por un simulador de selección. El simulador, estaba compuesto por una serie de zarandas, una puesta sobre la otra, doce en total, que permitía el paso de los distintos tamaños relativos y tipos de grano (plano y redondo). El simulador, adicionalmente estaba equipado con un motor que permitía accionarlo y producir un movimiento vibratorio permitiendo el movimiento de la semilla y su flujo a través de las zarandas.

Luego de depositar el kilogramo de semilla en el simulador, éste se accionó produciendo un movimiento vibratorio, depositando los granos en cada zaranda dependiendo su tamaño y tipo. Posteriormente se procedió a determinar el tipo de grano (plano o redondo) y el número de éstos. El rendimiento de semilla estuvo determinado su peso, provenientes de cada parcela útil. Los pesos fueron corregidos por población, uniformizados al $15 \%$ de humedad y convertidos a kilogramos por hectárea mediante la siguiente ecuación (Ec.1).

$$
\text { Kg. ha } a^{-1}=\frac{10000 * R P *(100-H C) *(P i-03 * P F}{85 * P C * A P} \quad \text { (EC. 1). }
$$

Donde:

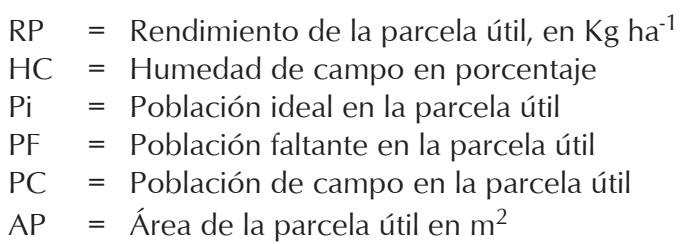

\section{Análisis de datos}

Los datos generados se analizaron usando el programa estadístico SAS (SAS Institute Inc, 1997). Se empleó la prueba de comparación de medias Tukey a un nivel de significancia $P<0.05$. En el análisis, sólo se usaron aquellas variables en las cuales el análisis de varianza mostró diferencias estadísticamente significativas a un nivel de significancia $\mathrm{P}<0.05$.

\section{Resultados y Discusión}

Según el análisis preliminar mediante el análisis de varianza, al menos uno de los siete tratamientos evaluados presentó comportamiento diferencial respecto a los demás, los cuadrados medios obtenidos en el análisis de varianza permiten concluir que la mayor variación se debe a los tratamientos y que el aporte de éstos a la variación total del modelo estadístico es del $84 \%$. El porcentaje restante (16\%), es atribuido a los factores de bloqueo (Tabla 1). Los datos analizados presentaron un $\mathrm{R}$ cuadrado de 0.71 y un coeficiente de variación de $8.97 \%$. El valor medio de rendimiento fue de 3.97 toneladas de semilla por hectárea.

Tabla 1. Cuadrados medios y significancia estadística para la variable rendimiento per se de la línea progenitora materna evaluada.

\begin{tabular}{|l|c|c|c|c|c|}
\hline \multicolumn{1}{|c|}{ FV } & GL & SC & CM & F & Pr $>$ F \\
\hline Bloque & 6 & 1.850 & 0.308 & 2.42 & 0.0453 \\
\hline Tratamiento & 6 & 9.375 & 1.562 & 12.28 & $<0.0001$ \\
\hline Error & 36 & 4.579 & 0.127 & & \\
\hline
\end{tabular}

Para la variable rendimiento per se de la línea evaluada se encontraron diferencias estadísticamente significativas entre los tratamientos, según la prueba de comparación de medias de Tukey a un nivel de significancia $\mathrm{P}<0.05$.

Al realizar la prueba de comparación de medias en cada uno de los tratamientos evaluados (Figura 1), se encontró que el rendimiento tiene comportamientos extremos al no eliminar la panoja de la planta, por ende, no hay pérdida de hojas y al eliminar la panoja más las cinco hojas por debajo de ésta. Con el tratamiento dónde no pérdida de la panoja y hojas (1), se obtuvieron estadísticamente los mayores rendimientos (4.83 ton ha-1), y para el caso extremo donde hubo la pérdida de las cinco hojas y la panoja (7), el rendimiento obtenido fue el menor, con una media de 3.3 ton ha ${ }^{-1}$, equivalente a $31.7 \%$ menos. Entre éstos dos tratamientos hubo diferencias estadísticamente significativas $(\mathrm{P}<0.05)$.

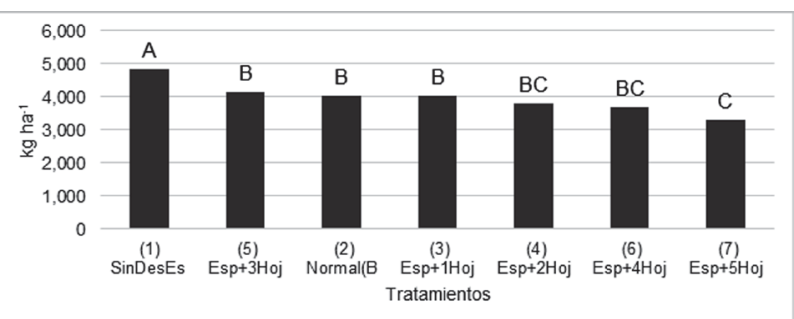

DMS: 58.32 Kilogramos, ${ }^{*}$ Medias con la misma letra no son significativamente distintas $\mathrm{P}<0.05$. Prueba de comparación de Medias Tukey.

Figura 1. Comparación de medias para la variable rendimiento en una línea progenitora de un híbrido de maíz comercial en los diferentes tratamientos, testigo sin eliminar panoja y despanojado con diferente número de eliminación de hojas. 
En los demás tratamientos, la eliminación de la panoja incluidas 1, 2, 3 o 4 hojas y la eliminación de la panoja por el método tradicional, donde la pérdida de hojas es variable, en promedio tres, se encontró que no hay diferencias estadísticamente significativas que afecten el rendimiento. No obstante, el menor rendimiento entre los tratamientos se obtuvo al eliminar la panoja y cuatro hojas. El mayor rendimiento fue el obtenido cuando se perdían tres hojas y la panoja.

Los resultados para la variable frecuencia de granos de la semilla F1 comercial, teniendo en cuenta los distintos calibres y tipos comerciales (planos y redondos), indicaron que se aceptan granos de tipo plano y redondo, éstos de igual modo se comercializan en distintos calibres, desde el 22 hasta el 16. No obstante, los calibres de mayor aceptación en el mercado son: el 22, 20 y 18. Esto debido a que es semilla de grano más grande y facilita su manejo en el momento que los agricultores realizan las siembras.

Es importante destacar que semillas de calibres más pequeños representan menor peso con relación a las semillas que puede contener una presentación comercial. Estas presentaciones generalmente son de 60,000 semillas o, en su defecto, de 20 kilogramos. En términos generales, una bolsa de 60,000 semillas de calibre 22 pesará más si es comparada con una bolsa de 60,000 semillas de un calibre inferior.

Para el análisis de los granos de tipo planos calibre 22 (Figura 2), no se encontró diferencias estadísticamente significativas entre los tratamientos evaluados. Sin embargo, el tratamiento número 7 (Panoja+5Hojas) fue el que produjo menos en términos de frecuencia o número de granos, la disminución fue del 51.2\%. Este dato se puede relacionar con el rendimiento per se de la línea, ya que mayor pérdida de hojas influye negativamente en el rendimiento. La formación de granos

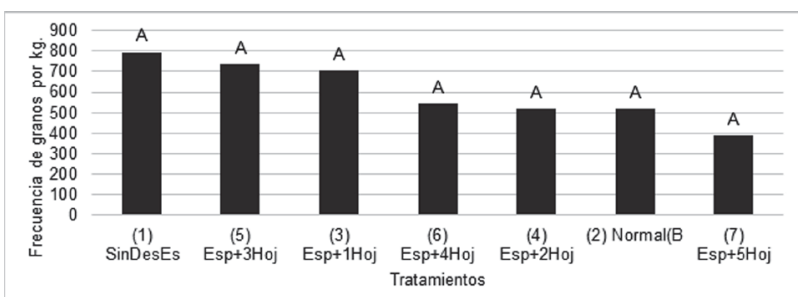

\footnotetext{
**Medias con la misma letra no son significativamente distintas $(P<0.05)$.

Figura 2. Comparación de medias para la variable frecuencia de semilla F1 comercial calibre 22 Plano en una línea progenitora de un híbrido de maíz comercial en los diferentes tratamientos, testigo sin eliminar panoja y despanojado con diferente número de eliminación de hojas. DMS: 525.14 granos.
}

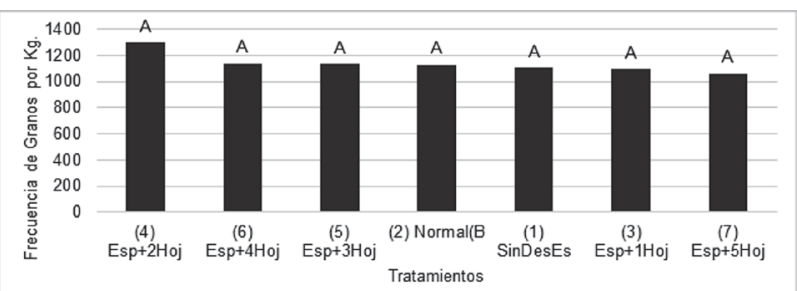

DMS: 465.23 granos. ${ }^{*}$ Medias con la misma letra no son significativamente distintas $(\mathrm{P}<0.05)$.

Figura 3. Comparación de medias para la variable frecuencia de semilla F1 comercial calibre 20 Plano en una línea progenitora de un híbrido de maíz comercial en los diferentes tratamientos, testigo sin eliminar panoja y despanojado con diferente número de eliminación de hojas.

por mazorca es menor y el espacio entre ellos aumenta permitiendo que éstos, en su fase de llenado, se ensanchen posibilitando que la frecuencia de granos redondos sea mayor. Este resultado es congruente a los datos reportados para los tamaños de semilla 20 Redondo (Figura 6), donde la frecuencia de grano de tipo redondo es mayor cuando se pierde el mayor número de hojas (Esp+5Hojas) tratamiento 7.

Para los granos de los tamaños 20 y 18 planos (Figura 3 y 4), la frecuencia de granos de tipo plano no presentó diferencias estadísticamente significativas. Todos los tratamientos fueron estadísticamente iguales para granos planos calibres 20 y 18.

Respecto al tipo de grano redondo (Figuras 5 y 6), la frecuencia para los calibres 22 y 20 no presentó diferencias estadísticamente significativas. No obstante, las frecuencias más altas de este tamaño de grano para el calibre 20, sin ser estadísticamente diferente de las demás, se obtuvieron en la evaluación del tratamiento número 7(Esp+5Hojas), como se muestra en la Figura 6.

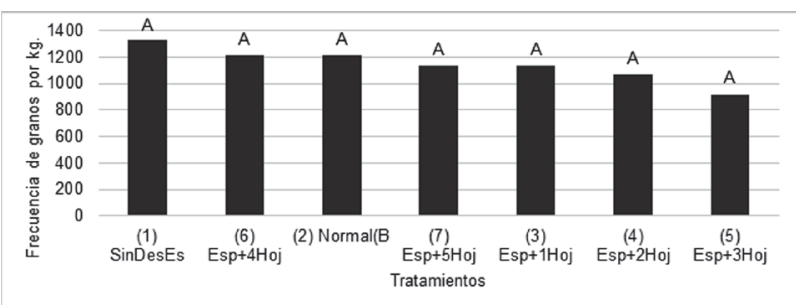

DMS: 503.54 granos. ${ }^{*}$ Medias con la misma letra no son significativamente distintas $(P<0.05)$.

Figura 4. Comparación de medias para la variable frecuencia de semilla F1 comercial calibre 18 Plano en Comparación de medias para la variable rendimiento en una línea progenitora de un híbrido de maíz comercial en los diferentes tratamientos, testigo sin eliminar panoja y despanojado con diferente número de eliminación de hojas. 
Estadísticamente, los granos redondos en todos los tratamientos evaluados, presentaron la misma frecuencia.

Los resultados obtenidos respecto a los granos de tipo redondo calibre número 20 (Figura 6), permiten suponer que la pérdida de hojas influye negativamente en la tasa de fecundación efectiva en la mazorca, lo que se traduce en una menor cantidad de granos formados, lo que permite concluir que a menor cantidad de granos formados en la inflorescencia femenina aumenta la frecuencia de granos redondos.

El menor tamaño de granos de tipo redondo (calibre 18), presentó diferencias estadísticas entre los tratamientos evaluados. La mayor frecuencia se presentó en la media del tratamiento 7 (Figura 7).

El tratamiento número 1 sin despanojar (SinDesEs), fue significativamente diferente a los demás tratamientos, presentando la menor frecuencia de granos redondos (Figura 7). Esta variación es atribuida a que el llenado de la inflorescencia femenina (mazorca) es mejor y está relacionado con la no pérdida de hojas y panoja. Fisiológicamente, la planta no sufre ningún estrés que interfiera en su normal desarrollo para la formación de granos, por lo tanto, en el raquis se forma el mayor número de granos permitiendo que éstos en su mayor frecuencia sean todos planos, debido a los pocos o nulos espacios que quedan entre éstos evitando su ensanchamiento y tendencia a la formación de granos redondos.

\section{Discusión}

La información obtenida con el presente estudio, teniendo en cuenta cruzamientos simples para la formación del híbrido SEM1430, tiene gran relevancia para las condiciones agroclimáticas del Valle del Cauca -

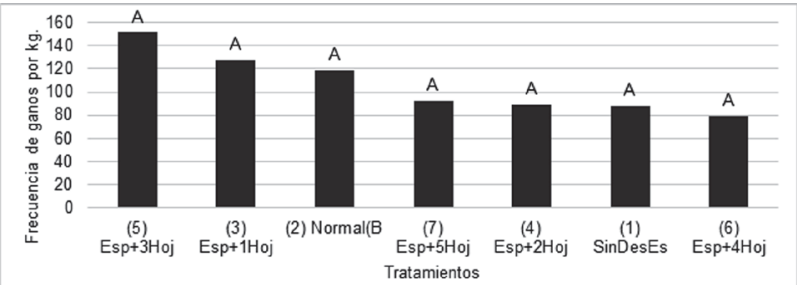

DMS: 110.7 granos. ${ }^{*}$ Medias con la misma letra no son significativamente distintas $(P<0.05)$.

Figura 5. Comparación de medias para la variable frecuencia de semilla F1 comercial calibre 22 Redondo Comparación de medias para la variable rendimiento en una línea progenitora de un híbrido de maíz comercial en los diferentes tratamientos, testigo sin eliminar panoja y despanojado con diferente número de eliminación de hojas.

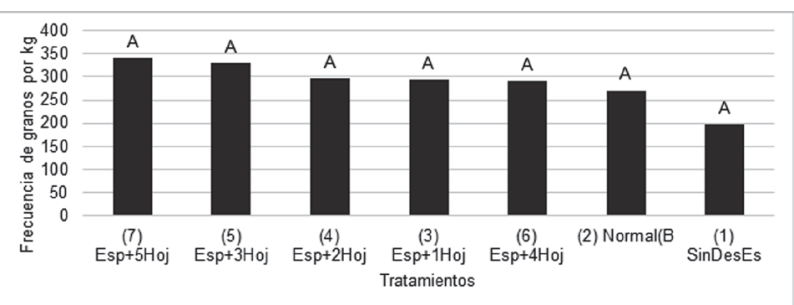

DMS: 197.64 granos. * Medias con la misma letra no son significativamente distintas al $(\mathrm{P}<0.05)$.

Figura 6. Comparación de medias para la variable frecuencia de semilla F1 comercial calibre 20 Redondo en los diferentes tratamientos.

Colombia, debido a que permite concluir que, aún eliminando tres hojas junto con la panoja, el rendimiento de semilla se mantiene en niveles aceptables comercialmente. Sin embargo, como es de esperarse, las proporciones en la frecuencia de tamaños de semilla de los distintos calibres de interés comercial poseen variaciones, sin que éstas a nivel experimental tengan relevancia estadística.

Estas variaciones son congruentes con lo afirmado por Ruiz-Cerda, (1988), quien reporta que al eliminar la panoja se reduce la dominancia apical, produciendo cambios favorables en el balance hormonal de la planta, que repercuten en el crecimiento y desarrollo de la inflorescencia femenina; refuerza los resultados obtenidos por Obregon, et al., (1969), Espinosa-Calderon, et al., (1998) y que son similares a los reportados en este estudio. Estos autores demostraron que los mayores rendimientos se obtuvieron cuando la planta no perdía la panoja ni sus hojas y que los menores resultados se expresaban cuando había pérdida del mayor número de hojas. Los rendimientos intermedios se obtuvieron al eliminar de la planta la panoja más 1 a 4 hojas.

Adicionalmente, las observaciones y resultados obtenidos del presente trabajo refuerzan las planteadas

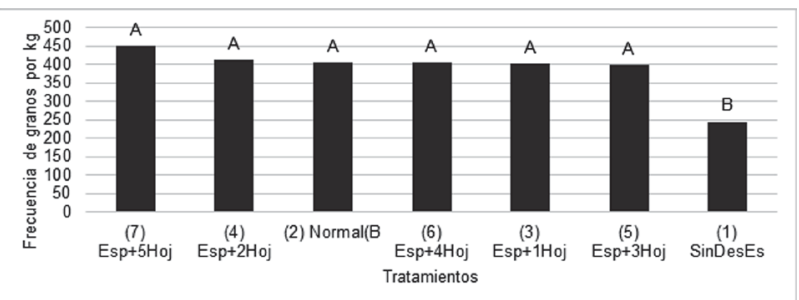

DMS: 155.48 granos,. ${ }^{* *}$ Medias con la misma letra no son significativamente distintas al $(\mathrm{P}<0.05)$.

Figura 7. Comparación de medias para la variable frecuencia de semilla F1 comercial calibre 18 Redondo en los diferentes tratamientos. 
por Espinosa-Calderón, et al., (1998), quienes además plantean que, con la intención de asegurar buena calidad en el despanojado, evitando autofecundaciones y con menor inversión de jornales, podría sugerirse que la práctica de eliminación de la panoja sea realizada eliminando una y hasta tres hojas, lo que facilita esta labor.

Los resultados obtenidos, fueron resultado de la evaluación de los progenitores de un híbrido de cruza simple y bajo condiciones climáticas del Valle del Cauca - Colombia. En la búsqueda de maximizar la operatividad y reducir los costos al momento de producción de semilla híbrida de maíz, se hace necesario la evaluación de diversos híbridos para corroborar que los resultados aquí obtenidos se pueden aplicar a una estrategia de despanojado mecánico.

\section{Conclusiones}

La pérdida de hojas cercanas a la panoja de la planta indiscutiblemente afecta fisiológicamente su desarrollo y su rendimiento. No obstante, la pérdida de 1 a 4 hojas en el proceso de despanojado no representa diferencias estadísticamente significativas en el rendimiento, siendo éste el número de hojas que generalmente se pierden al despanojar lotes de maíz con fines de producción de semilla hibrida comercial.

Las frecuencias del tamaño de las semillas de tipo comercial no se ven afectadas, pues la cantidad de semilla de tipo plano y redondo en sus distintos calibres (tamaño relativo) fueron estadísticamente iguales en todos los tratamientos evaluados.

La mayor pérdida de hojas en el despanojado favorece la formación de granos redondos en la mazorca, aumentando su frecuencia, pero disminuyendo el rendimiento per se de la línea evaluada, y la mayor frecuencia de granos planos en la inflorescencia está asociada a menor pérdida de hojas en el despanojado.

\section{Referencias}

Agrios G. (2012). Plant pathology (5 ed.). Burlington: Academic Press.

Centro de Investigación de la Caña de Azúcar de Colombia. (2013). http://www.cenicana.org/. Retrieved Marzo 10, 2014, from http://www.cenicana.org/clima_/index.php\#

Espinosa-Calderón A, Tadeo-Robledo M. Desespigamiento en cruzas simples de maíz y su efecto en la producción de semillas. Rev Fitotec Mex. 1995;18(1):9-15.

Espinosa-Calderón A, Tadeo-Robledo M. Evaluación de desespigue mecánico en híbridos dobles de maíz en los Valles Altos de México. Agronomía Mesoamericana, 1998;9(1):90-92.

Espinosa-Calderón A, Ortiz-Careceres J, Ramírez-Fonseca A, GómezMontiel NO, Martínez-Garza A. Productividad de semilla de líneas tropicales de maíz (Zea mays L.) del CIMMYT e INIFAP. Agricultura Técnica en México. 1999;25(1):53-58.

Espinosa-Calderón A, Tadeo-Robledo M, Meza-Guzmán LD, Arteaga-Escamilla I, Matías-Buatista D, Valdivia-Bernal R, ZamudioGonzález B. Eliminación de espiga y hojas en un híbrido de maíz androestéril y fértil. Universidad y Ciencia Trópico Húmedo. 2010;26(3):215-224.

Espinosa-Calderón A, Tadeo-Robledo M, Sierra-Macías M, CaballeroHernández F, Valdivia-Bernal R, Gómez-Montiel NO. Despanojado y densidad de población en una cruza simple androestéril y fértil de maíz. Agronomía Mesoamericana. 2010;21(1):159165.

García-Montealegre JP. (2008). Manejo eficiente de nutrientes en el cultivo del maíz en Colombia. Bogotá: Fenalce. Pag 126.

Obregon P, Maneiro JM. Influencia de la pérdida de hojas en el despanojado sobre el rendimiento del maíz. Agronomía Tropical. $1969 ; 19(3): 205-210$.

Ruiz-Cerda E. (1988). Respuesta de tres genotipos de maíz (Zea mays L.) al desespigamiento y densidad de población: Tesis de Maestro en Ciencias en Producción Agrícola Universidad Autónoma de Nuevo León. Monterrey, México.

SAS Institute Inc. (1997). SAS/STAT Software: Changes and enhancenments throgh release 6.12 . Cary, N C, USA. Institute Inc.

Tadeo-Robledo M, Espinosa-Calderón A, Solano AM, Martínez R. Androesterilidad en líneas e híbridos de maíz de Valles Altos de México. Agronomía Mesoamericana. 2003;14(1):15-19. 